\title{
Contribution à l'étude des jaunisses de la vigne dans le monde. Prospection par test Elisa spécifique du mycoplasma-like organism (MLO) de la flavescence dorée
}

\author{
C Kuszala 1*, O Cazelles 2, J Boulud 3, R Credi 4, G Granata 5, G Kriel 6, P Magarey 7, \\ C Magnien 3, RC Pearson 8, E Refatti 9, E Tanne 10, A Caudwell 1
}

\footnotetext{
1 INRA, station de recherches sur les mycoplasmes et les arbovirus des plantes, BV 1540, F21034 Dijon, France;

2 Station fédérale de recherches agronomiques de Changins, $\mathrm{CH}-1260$ Nyon, Suisse;

3 Service régional de la protection des végétaux de Bourgogne, ZI Nord, BP 177, F21205 Beaune cedex, France;

4 Istituto di Patologia Vegetale dell'Universita, via Filippo Re 8, 140126 Bologna;

5 Universita degli Studi di Catania, Istituto di Patologia vegetale, via Valdisavoia 5, Catania, Sicilia, Italie;

6 KWV, PO Box 528, Suider Paarl 7624, Union Sud-africaine;

7 Department of Agriculture, Loxton Research Center, PO Box 411, Loxton, South Australia 5333, Australie;

${ }^{8}$ Department of Plant Pathology, Cornell University, New York State Agricultural Experiment Station, Geneva, New York 14456-0462, États-Unis;

9 Universita di Udine, Dipartimento di Biologia applicata alla Difesa delle Plante, viale delle Science, 208 (Localita Rizzi), 133100 Udine, Italie;

10 Agricultural Research Organization, The Volcani Center Institute of Plant Protection, Pob 6, Bet Dagan 50250, Israël
}

(Reçu le 13 juillet 1993; accepté le 21 septembre 1993)

\begin{abstract}
Résumé - Les jaunisses de la vigne réunissent un ensemble de maladies dont les symptômes sont identiques. La mise au point récente d'un test Elisa spécifique de la flavescence dorée (FD) sur échantillon de vigne a permis de différencier la FD des autres jaunisses. Ce test permet de détecter la FD sur différents cépages, au vignoble, et sur des porte-greffes, en serre, présentant de légers symptômes. Il a été appliqué à des échantillons de vigne provenant de régions différentes du monde entier. La FD a été localisée ainsi dans le Sud de la France et le Nord de l'Italie (Friule). En France, ces résultats ont permis de clarifier la situation très confuse concernant la présence de la FD et du bois noir en Bourgogne, ainsi que dans les régions voisines. Les échantillons de Suisse, d'Italie (Émilie romagne et Sicile), Australie, Israël, États-Unis (État de New York), ainsi que ceux de l'Union Sud-Africaine infectés par la maladie du Shiraz, ont réagi négativement. Ces jaunisses n'ont donc pas de relation sérologique avec la FD. Une telle enquête ne garantit pas l'absence de la FD dans les régions prospectées, cependant, elle permet de mettre en évidence l'importance des autres jaunisses.
\end{abstract}

jaunisses de la vigne / Elisa / flavescence dorée / MLO

Summary - Contribution to the study of grapevine yellows in the world: investigation by Elisa using flavescence dorée-specific antibodies. Grapevine yellows (GY) are a group of diseases with similar symptoms. The recent publication on the use of Elisa to detect the flavescence dorée (FD) mycoplasma-like organism (MLO) in the grapevine itself has allowed the differentiation of FD from the other GY. In order to investigate the epidemiology of FD, grapevine canes or leaves showing GY symptoms were harvested from several areas in the world (France, 138 samples; Northern Italy and Sicily, 13; Switzerland, 39; Israel, 9; North America, 31; South Africa, 24; and Australia, 26) and tested by Elisa using specific polyclonal and monoclonal antibodies raised against FD. FD-MLO were specifically detected in various cultivars from the field and in rootstock cuttings grown in the greenhouse and showing mild symptoms. In this work, FD was found to be confined to grapevine stocks in 2 regions of Europe: southern France and

* Correspondance et tirés à part.

Abréviations : BN : bois noir ; CHAPS : 3 - [ (3-cholamidopropyl) diméthylammoniol] -1-propanesulfonate ; Elisa : enzyme-linked immunosorbent assay; FD : flavescence dorée; MLO : mycoplasma-like organism. 
northern Italy (Friuli). In France, these results have allowed clarification of a very confusing situation concerning FD and Bois noir $(B N)$. All the symptomatic vines of north-east France reacted negatively, and hence probably belong to $B N$. Some samples from the south of the Rhône valley (Ardèche) reacted similarly. No positive FD reaction was obtained with samples showing GY symptoms from Sicily, Emilia Romagna (Italy), Switzerland (western part and Tessin), Israel, USA (New York State), South Australia or with Shiraz decline desease from the Republic of South Africa. These results suggest that no serological relationship exists between FD-MLO and the other GY. However this study does not exclude that FD could be present in some parts of these areas. This work shows the economic importance of the GY other than FD, the risk of epidemics and the necessity to promote protecion, cure and eventual quarantine measures.

grapevine yellows / Elisa / flavescence dorée / MLO

\section{INTRODUCTION}

Confrontés au développement des jaunisses de la vigne dans le monde et au caractère parfois très grave de ces maladies, nous avons entrepris une prospection internationale. Le terme de jaunisse a été donné pour souligner le symptôme le plus frappant de ces maladies, bien que sur les cépages rouges on observe au contraire un rougissement. Ce changement de coloration est dû à un blocage du liber qui se nécrose. Dans ce groupe se trouvent, entre autres, la flavescence dorée (FD) et la maladie du bois noir (BN) (Caudwell et al, 1971). La FD est bien caractérisée par sa forme épidémique, transmise par la cicadelle Scaphoideus titanus Ball et par son agent pathogène, un MLO (Mycoplasma-like organism) non cultivable in vitro. Bien que son diagnostic dans un vignoble débute par la recherche d'une association de symptômes de "type FD" et de son vecteur (le seul actuellement connu), le diagnostic de la FD, par simple visualisation de symptômes, reste aléatoire en raison de sa similitude avec les autres jaunisses, et de la superposition possible de jaunisses différentes dans une même région. Le BN est endémique et on ne connaît pas son mode de propagation. Par le caractère fluctuant de l'apparition de ses symptômes d'une année sur l'autre, il est difficile à étudier. II se distingue de la FD par le fait que la cicadelle vectrice de la FD ne transmet pas le BN, même expérimentalement (Caudwell et al, 1971). Le syndrome de la FD, défini en France (Caudwell et Ottenwaelter, 1957), a été retrouvé dans les vignobles de différents pays du monde (Tanne et Mitzany 1973; Credi et Babini, 1984 ; Belli et al,1985 ; Granata, 1985 ; Pearson et al,1985; Rumbos et Avgelis, 1985 ; Magarey et Wachtel, 1986 ; Cazelles et al,1992).

En France, le nombre des vignobles présentant des symptômes de jaunisse semble en progression, plus particulièrement en Bourgogne et dans les régions voisines. Une situation assez confuse semblait s'y être installée, la FD prenant pied dans une zone où le BN était déjà présent.

En Suisse romande, des ceps isolés montrent des symptômes de jaunisse en l'absence de la cicadelle Scaphoideus titanus Ball (Cazelles et al, 1992). En Italie, l'épidémie d'une jaunisse s'est développée dans les provinces du Sud et en Sicile (Granata, 1982) : des MLO furent observés en microscopie électronique en relation avec les symptômes (Granata et Grimaldi, 1991) et en absence du vecteur de la FD. Au Nord de I'Italie, des symptômes de jaunisse furent trouvés en présence de Scaphoideus titanus Ball (Belli et al, 1984). En Israël, dès 1973, Tanne et Mitzany ont observé des symptômes de jaunisses sur Chardonnay. Aux États-Unis, Pearson et al (1985) ont signalé la présence d'une jaunisse à symptômes semblables à la FD. En Australie, la maladie est épidémique dans certaines régions et endémique dans d'autres (Magarey et Wachtel,1986). En Afrique du Sud, Engelbrecht et Kasdorf (1990) ont mis en évidence la présence d'un clostérovirus en relation avec la maladie de Shiraz dont les symptômes sont voisins de ceux des jaunisses.

Il nous a paru intéressant de vérifier si certaines de ces jaunisses étaient dues au MLO de la FD, ou éventuellement si elles avaient une relation sérologique avec celui-ci. La mise au point par Caudwell et Kuszala en 1992 d'un test Elisa spécifique de la FD sur vigne permet d'en faire l'étude.

\section{MATÉRIEL ET MÉTHODES}

\section{Matériel végétal}

Les échantillons provenaient de différentes régions de France (Alsace, Ardèche, Aude, Bourgogne, Champagne et Franche-Comté), d'Italie (Nord et Sicile) et de différents pays: Suisse, Israël, sud de l'Australie, 
États-Unis (État de New York) et Afrique du Sud. En France, ils ont été choisis dans les foyers qui avaient le pourcentage de pieds malades le plus élevé dans le vignoble. Certains ceps présentaient les symptômes de la maladie depuis plusieurs années consécutives. L'enquête a porté sur différents cépages, en particulier le Chardonnay, cépage réputé très sensible à la FD. Ces vignes présentaient les symptômes typiques de la FD :

- sur feuillage : enroulement accompagné de jaunissement ou de rougissement du limbe, soit généralisé, soit sectoriel limité par les nervures ;

- sur bois : non-aoûtement des sarments de la base au sommet ;

- sur grappes : dessèchement de la rafle et des grains.

Les échantillons ont été récoltés soit sous forme de rameaux, soit sous forme de feuilles de vigne présentant les symptômes nets de la FD. Ils ont été lavés à l'eau permutée, et conditionnés individuellement en sachets plastiques scellés puis congelés à $-20^{\circ} \mathrm{C}$ en attendant d'être testés. Certains échantillons ont été lyophilisés.

\section{Préparation des antigènes pour l'Elisa}

La méthode utilisée consiste à broyer à l'aide d'un broyeur à billes (Gugerli, 1984) $1 \mathrm{~g}$ de feuilles sans le pétiole, dans $10 \mathrm{ml}$ d'un tampon contenant $5 \%$ du détergent CHAPS (Caudwell et Kuszala, 1992).

Pour amplifier la sensibilité du test, nous avons procédé, dans la majorité des cas, à la concentration (10X) de l'antigène par filtration moléculaire dans les concentrateurs Centrisart Sartorius : seuil de coupure $20000 \mathrm{Da}$.

Des échantillons témoins de vignes saines et malades (FD) en provenance de l'Aude (France) ont été traités de façon identique aux échantillons à tester.

\section{Le test Elisa}

Les comparaisons ont été faites selon la méthode Elisa Sandwich (Boudon-Padieu et al, 1989 ; Schwartz et al,1989). Le test comporte 5 étapes :

- des anticorps polyclonaux de lapin anti-fève FD (origine INRA Dijon : $3,5 \mu \mathrm{g} / \mathrm{ml}$ ) sont adsorbés sur une plaque de microtitration (Nunc Immuno plate Maxisorp) pendant $2 \mathrm{~h}$;

- les échantillons sont déposés et mis en incubation une nuit à $4^{\circ} \mathrm{C}$;

- un mélange de 3 ou 4 anticorps monoclonaux antiFD (origine INRA Dijon : FD1, 51 et 113 ou FD12, 17, 18 et 51) prélevés dans les surnageants de culture d'hybridomes est déposé pendant $2 \mathrm{~h}$ (le mélange des 4 s'est révélé très supérieur au mélange 51,113 et FD1, qui avait été utilisé dans la mise au point de la méthode) ;
- des anticorps de chèvre antisouris conjugués à la phosphatase alcaline (Biosys Bl2513CE/73) sont déposés sur la plaque pendant $2 \mathrm{~h}$;

- la révélation se fait avec le paranitrophénylphosphate (PNPP) à $1 \mathrm{mg} / \mathrm{ml}$ dans du tampon diéthanolamine $\mathrm{pH} 9,8$. On mesure les différences d'absorption aux 2 longueurs d'onde $405 \mathrm{~nm}$ et $490 \mathrm{~nm}$ avec un spectrophotomètre Molecular Device. Les résultats sont exprimés en densité optique (DO). On admet comme positifs les résultats où la DO de l'échantillon malade est supérieure à 2 fois celle de l'échantillon sain pour un temps d'incubation de substrat de $2 \mathrm{~h} 30$.

\section{RÉSULTATS}

Le test permet de détecter la FD sur différents cépages en plein champ (Alicante, Carignan, Garganeva, Chardonnay, Grenache, Perera, Pinot noir, Sauvignon) et en serre (Baco 22A, Ugni blanc), ainsi que sur des porte-greffes présentant de légers symptômes en serre : Fercal, $1103 \mathrm{P}$ et $3309 \mathrm{C}$ (tableau I). Nous constatons surtout que la FD est ainsi détectée dans le sud de la France et en Italie dans la région de Friule. En revanche, elle n'est pas décelée dans le nord-est de la France (Daire et al, 1993a), la Suisse (Cazelles et Kuszala, 1993) et I'Italie (région de Bologne et Sicile). Les résultats des tests Elisa des échantillons provenant du sud de l'Australie, d'Israël, d'Afrique du Sud (maladie du Shiraz) et ceux des États-Unis ne réagissent pas en présence des anticorps spécifiques de la FD qu'ils aient été concentrés ou non (tableau II).

Nous pouvons dire que les vignes malades, prélevées dans les foyers étudiés et répondant négativement au test Elisa, ne sont pas atteintes de la FD et n'ont pas de relation sérologique avec le MLO de la FD. Nous pensons qu'en France il pourrait s'agir d'une autre jaunisse, comme le bois noir (BN) ou la Vergilbungskrankheit. Cette maladie est très semblable à la FD et se rencontre en Allemagne sur le Riesling dans les vallées du Rhin et de la Moselle (Gärtel, 1959). Mais actuellement nous manquons de test sérologique spécifique pour vérifier cette hypothèse.

\section{DISCUSSION ET CONCLUSION}

En France, plus particulièrement en Bourgogne et dans les régions voisines, ces résultats ont permis de clarifier la situation très confuse résul- 
Tableau I. Bilan des tests sérologiques Elisa spécifiques de la FD de la vigne sur des échantillons concentrés ou non 10 fois, en provenance de différents pays d'Europe (résultats; - négatif; + et ++ : positifs). 1 : extrait non concentré ; 2 : extrait concentré 10X. a) Caudwell A, Kuszala C, 1992 ; b) Daire X et al, 1993 ; c) Cazelles O, Kuszala C, 1993 ; d) échantillons apportés par G Granata; e) échantillons envoyés par $R$ Credi ; f) Kuszala $C$ (matériel de serre ou de pépinière) ; g) échantillons envoyés par $E$ Refatti.

\begin{tabular}{lll}
\hline Pays Cépages & $\begin{array}{l}\text { Elisa sur Réf } \\
\text { extraits } \\
\end{array}$ & $1 \quad 2$ \\
\hline
\end{tabular}

\section{France}

Région nord-est (Alsace, Ardèche, Bourgogne, Champagne, Franche-Comté)

$\begin{array}{cll}94 \text { ceps } & \text { Chardonnay } \\ 10 " & \text { Gamay } \\ 1 \text { " } & \text { Gewurztraminer } \\ 4 \text { " } & \text { Pinot Noir } \\ 3 \text { " } & \text { Riesling } \\ 1 \text { " } & \text { Savagnin } \\ 1 \text { " } & \text { Tokay } \\ 4 \text { " } & \text { Trousseau }\end{array}$

Aude et sud de la France

$\begin{array}{lll}8 \text { ceps } & \text { Alicante } \\ 1 \text { " } & \text { Baco 22A } \\ 2 " & \text { Carignan } \\ 2 " & \text { Chardonnay } \\ 2 " & \text { Fercal } \\ 3 \text { " } & \text { Grenache } \\ 3 & \text { " } & \text { Pinot Noir } \\ 1 " & \text { Ugni Blanc } \\ 1 " & 3309 \mathrm{C} \\ 1 " & 1103 \mathrm{P}\end{array}$

Suisse

37 ceps Chardonnay

2 " Gamay

Italie

\begin{tabular}{|c|c|c|c|c|}
\hline \multicolumn{4}{|l|}{ Bologne } & e \\
\hline 3 ceps & Caveccia & - & - & \\
\hline $2 "$ & Sangiovese & - & - & \\
\hline Friule & & & & $\mathrm{g}$ \\
\hline 1 cep & Chardonnay & + & ++ & \\
\hline $1 "$ & Garganeva & + & ++ & \\
\hline 1 & Perera & + & ++ & \\
\hline 1 & Sauvignon & + & ++ & \\
\hline Sicile & & & & d \\
\hline 5 ceps & Chadonnay & - & - & \\
\hline $6 "$ & Inzolia & - & - & \\
\hline $1 "$ & Nerello & - & - & \\
\hline $1 "$ & Perricone & - & - & \\
\hline
\end{tabular}

Tableau II. Bilan des tests sérologiques Elisa spécifiques de la FD sur des échantillons de vignes, concentrés on non 10 fois, en provenance de différents pays du monde (excepté l'Europe) (résultats; - négatif). 1 : extrait non concentré; 2 : extrait concentré $10 \mathrm{X}$. Échantillons envoyés par : h) Magarey $P$; i) Tanne $E$; j) Kriel G ; k) Pearson RC.

\begin{tabular}{|c|c|c|c|c|}
\hline \multirow[t]{2}{*}{ Pays } & \multirow[t]{2}{*}{ Cépages } & \multicolumn{2}{|c|}{$\begin{array}{l}\text { Elisa sur } \\
\text { extraits }\end{array}$} & \multirow[t]{2}{*}{ Réf } \\
\hline & & 1 & 2 & \\
\hline \multicolumn{4}{|c|}{ Australie } & $\mathrm{h}$ \\
\hline 26 ceps & Divers & - & - & \\
\hline \multicolumn{4}{|l|}{ Israël } & $\mathrm{i}$ \\
\hline 9 ceps & Chardonnay & - & - & \\
\hline \multicolumn{4}{|c|}{ Union Sud-Africaine } & j \\
\hline 2 ceps & Cabernet Sauvignon & - & - & \\
\hline 2 ceps & Cinsaut Noir & - & - & \\
\hline 6 ceps & Ferdinand de Lesseps & - & - & \\
\hline 4 ceps & Malbec & - & - & \\
\hline 2 ceps & Merlot & - & - & \\
\hline 2 ceps & Ruby Cabernet & - & - & \\
\hline 1 ceps & Shiraz & - & - & \\
\hline 2 ceps & Weisser Riesling & - & - & \\
\hline 2 ceps & 99 Richter rootstock & - & - & \\
\hline \multicolumn{4}{|c|}{ États-Unis } & $\mathrm{k}$ \\
\hline 6 ceps & Chardonnay & - & - & \\
\hline $15 "$ & Riesling & - & - & \\
\hline $10 "$ & Vitis riparia & - & - & \\
\hline
\end{tabular}

tant de la similitude des symptômes de la FD et du BN. Les tests sérologiques négatifs pour la FD sont en accord en France avec quelques résultats obtenus par tests génomiques. En effet, ces tests génomiques FD spécifiques (Daire et al, 1992) montrent qu'il ne s'agit pas de la FD (Daire et al, 1993a). En revanche, la présence de la FD démontrée ici dans la région de Friule en Italie est directement confirmée par les tests génomiques réalisés sur les mêmes échantillons de vigne (Daire et al, 1993b). Elle est également en accord avec les tests sérologiques positifs pour la FD réalisés antérieurement sur des cicadelles Scaphoideus titanus utilisées pour vérifier leur caractère de vecteur naturel de la jaunisse de la vigne présente en Friule (Osler et al, 1992). Pour les autres échantillons, nous n'avons pas trouvé de relation sérologique avec la FD bien que, dans certains cas, comme en Sicile (Granata et Grimaldi, 1991), des MLO aient pu être observés en relation avec des symptômes de jau- 
nisse. Toutefois, une telle démarche ne garantit par l'absence totale de la FD dans les régions prospectées. Cependant, elle permet de mettre en évidence l'importance économique des autres jaunisses et la menace d'un risque latent dû à ces maladies inconnues.

\section{RÉFÉRENCES}

Belli G, Rui D, Fortusini A, Pizzoli L, Torresin G (1984) Presenza dell'insetto vettore (Scaphoideus titanus) e ulteriore diffusione della Flavescenza dorata nei vigneti del Veneto. Vignevini II, 9, 23-27

Belli G, Fortusini A, Rui D (1985) Recent spread of flavescence dorée and its vector in vineyards of Northern Italy. Phytopathol Mediterr 24, 189-191

Boudon-Padieu E, Larrue J, Caudwell A (1989) Elisa and Dot Blot detection of Flavescence dorée MLO in individual leafhopper vectors during latency and inoculative state. Curr Microb 19, 357-364

Caudwell A, Ottenwaelter M (1957) Deux années d'étude sur la flavescence dorée, nouvelle maladie grave de la vigne. Ann Amélior Plantes 7, 359-395

Caudwell A, Larrue J, Kuszala C, Bachelier JC (1971) Pluralité des jaunisses de la vigne. Ann Phytopathol 3, 1, 97-107

Caudwell A, Kuszala C (1992) Mise au point d'un test Elisa sur les tissus de vignes atteintes de flavescence dorée. Res Microbiol Inst Pasteur 143, 791 806

Cazelles O, Desbaillet C, Schmid A (1992) Jaunisses de la vigne en Suisse romande et au Tessin. Rev Suisse Vitic Arboric Hortic 24, 133-134

Cazelles O, Kuszala C (1993) Prospection des jaunisses de la vigne en Suisse romande et au Tessin et comparaison à la FD par le test Elisa. Rev Suisse Vitic Arboric Hortic 25, 4, 257-259

Credi R, Babini AR (1984) Cosi epidemici di Giallume della Vite in Emilia-Romagna. Vinevini 11, 35-39

Daire X, Boudon-Padieu E, Berville A, Schneider B, Caudwell A (1992) Cloned DNA probes for detection of grapevine Flavescence dorée mycoplasmalike organism (MLO). Ann Appl Biol 121, 95-103

Daire X, Kuszala C, Larrue J, Caudwell A, Magnien C, Boulud J (1993a) Les jaunisses de la vigne, flaves- cence dorée, bois noir, etc, en Bourgogne et dans les régions voisines. Progrès Agric Vitic $110 n^{\circ} 8$

Daire X, Clair D, Larrue J, Boudon-Padieu E, Alma A, Arzone A, Carraro L, Osler R, Refatti E, Granata G, Credi R, Tanne E, Pearson R, Caudwell A (1993b) Occurrence of diverse MLOs in tissues of grapevine affected by grapevine yellows in different countries. Vitis (sous presse)

Engelbrecht DJ, Kasdorf GGF (1990) Field spread of corky bark, fleck, leafroll and shiraz decline diseases and associated viruses in South African grapevines. Phytophylactica 22, 347-354

Gärtel W (1959) Die «flavescence dorée» oder "maladie du Baco 22A». Weinberg u Keller 6, 295311

Granata G (1982) Deperimenti e giallume in piante di vite. Inf Fitopatol 32 (7-8), 18-20

Granata G (1985) Epidemic Yellows in Vineyards of CV Insolia in Sicily. Phytopathol Mediterr 24, 79-81

Granata G, Grimaldi V (1991) Electron microscopic detection of mycoplasma-like organisms in epidemic yellow affected grapevines. Petria 1, 171-175

Gugerli P (1984) Une méthode simple pour le broyage de tissu végétal. Rev Suisse Vitic Arboric Hortic 16, 87-88

Magarey PA, Wachtel MF (1986) Australian grapevine yellows. Int J Trop Plant Dis 4, 1-14

Osler R, Boudon-Padieu E, Carraro L, Caudwell A, Refati $E$ (1992) First results on the trials in progress to identify the vector of the agent of a grapevine yellow in Italy. Phytopathol Mediterr 31, 175181

Pearson RC, Pool RM, Gonsalves D, Coffinet MC (1985) Occurrence of Flavescence dorée-like symptoms on "white Riesling" grapevines in New York, USA. Phytopathol Mediterr 24, 82-87

Rumbos IC, Avgelis AD (1985) Natural spread, importance and distribution of yellows, stem pitting, and enation disease of grapevine in some viticultural areas of Greece. Phytopathol Mediterr 24, 79-78

Schwartz Y, Boudon-Padieu E, Grange J, Meignoz R, Caudwell A (1989) Obtention d'anticorps monoclonaux spécifiques de l'agent pathogène de type mycoplasme de la flavescence dorée de la vigne. Res Microbiol 140, 311-324

Tanne E, Mitzany FE (1973) Virus diseases of grapevine in Israel. Vitis 12, 3, 222-225 